\title{
Iron as a promising alloying element for the cost reduction of titanium alloys: a review
}

\begin{abstract}
This article focuses on the effect of iron (Fe) addition on the fabrication of Ti-alloys. Fe is a potential inexpensive element that can be added to Ti-alloys to reduce their cost. This metal can also be used to replace expensive $\beta$-stabilizing alloying elements, such as vanadium (V) and molybdenum (Mo), for Ti-alloys. Fe has also been utilized as a novel cost-effective alloying element to decrease Ti-alloy costs and to design other alloys, such as Ti metal $62 \mathrm{~S}$ (Ti-6Al-1.7Fe-0.1Si) and Ti-Fe-O-N Ti-alloy. This technical perspective has been further applied to fabricate new Ti-alloys. For example, Ti8LC and Ti-5.5Al-1Fe with good mechanical features have been developed as novel Ti-alloys in China and Japan, respectively. Nowadays, vanadium (V) of Ti-6Al-4V alloy is completely replaced with Fe to produce TiAl-Fe alloy series. Three new alloys, namely, Ti-6Al-xFe, where $x=1,2$, and $3 \mathrm{wt} \%$, are introduced to examine the effect of $\mathrm{Fe}$ addition on the microstructure and mechanical properties of Ti-alloys.
\end{abstract}

Keyword: Cost reduction; Fe addition; Ti-alloy; $\beta$-stabilizing alloying elements 